\title{
Modeling the Energy Consumption of LoRaWAN in ns-3 Based on Real World Measurements
}

Conference Paper · October 2018

DOI: 10.1109/GIIS.2018.8635786

CITATIONS

3 authors, including:

Joseph Finnegan

National University of Ireland, Maynooth

9 PUBLICATIONS 31 CITATIONS

SEE PROFILE

Some of the authors of this publication are also working on these related projects:

Crosstalk Cancelation via Digital Pre-Distortion (DPD) View project

Methods to evaluate and enhance the performance of ultra-dense Wi-Fi Networks View project
READS

184

Ronan Farrell

National University of Ireland, Maynooth

191 PUBLICATIONS 680 CITATIONS

SEE PROFILE 


\section{Modeling the Energy Consumption of LoRaWAN in ns-3 Based on Real World Measurements}

\author{
Joseph Finnegan \\ Department of Computer Science \\ Maynooth University \\ Maynooth, Ireland \\ Email: joseph.finnegan@mu.ie
}

\author{
Stephen Brown \\ Department of Computer Science \\ Maynooth University \\ Maynooth, Ireland \\ Email: stephen.brown@mu.ie
}

\author{
Ronan Farrell \\ Department of Electronic Engineering \\ Maynooth University \\ Maynooth, Ireland \\ Email: ronan.farrell@mu.ie
}

\begin{abstract}
LPWAN technologies are defined by their focus on extended coverage while maintaining energy efficiency, at the expense of data throughput. In this research we enable the analysis of LoRa, a key LPWAN technology, in terms of energy efficiency. We perform real-world measurements of a standard LoRa chip and use the results to develop an energy consumption module in ns-3. Our contributions are an analysis of the energy consumption of different states in a LoRa transmission by the SX1272, the LoRa transceiver that is used in most common LoRaWAN devices, beyond what is provided in the datasheet, and an energy consumption module for use in three of the LoRaWAN ns-3 modules described in research, enabling more accurate energy consumption analysis of LoRa-based systems.
\end{abstract}

\section{INTRODUCTION}

The key defining attributes of LPWAN (Low Power Wide Area Networks) technologies are their focus on extended coverage while maintaining energy efficiency, at the expense of data throughput [1]. Therefore in order to properly evaluate an LPWAN technology it must be possible to analyse the technology in terms of energy efficiency. As the expected size of LPWA networks are with gateways expected to serve thousands of nodes, realistically it is desirable to be able to perform this analysis through simulation.

A key LPWAN technology is LoRa, which is a Frequency Shift Chirp Modulation scheme enabling long range, low data rate communication over the sub- $1 \mathrm{GHz}$ ISM bands. LoRaWAN defines an ALOHA-based MAC layer for a LoRabased wireless stack. Three devices classes are available in LoRaWAN: Class A (which supports device-initiated communication), Class B (Class A, with scheduled receive windows), and Class C (Class A, but always listening). A LoRaWAN library for Class A in ns-3 has been previously developed (separately by [2], [3], [4], [5]). In addition an analytical model for energy consumption of Class A has been developed by [6]. However currently there is no accurate method of modeling energy consumption of a LoRa transmission inside ns-3.

In this research we enable the analysis of LoRa in terms of energy efficiency by modeling the energy consumption of the SX1272, a standard LoRa chip. We perform real-world measurements of the chip to develop a device profile and use the results to create an energy consumption module in ns-3 to enable the evaluation of LoRa networks in terms of energy efficiency. Our modular code has been adapted to function as part of three of the LoRaWAN ns-3 modules that have been thus far described in research [2], [3], [4]. We evaluate our module through comparison to the theoretical limits computed through an analytical model [6] of the LoRaWAN protocol. Our results are comparable to those from the analytical model, showing both that our model has been correctly integrated into the externally-developed LoRaWAN module ([4]), and that [4] successfully simulates the LoRaWAN protocol.

This paper is outlined as follows: Section II describes related work. Section III describes the real world measurements performed. Section IV describes the resulting energy class developed from those results. Section V describes the simulations performed, and a comparison with the analyticallyderived results. Section VI concludes the paper.

\section{RELATED WORK}

\section{A. LoRaWAN Simulation Tools}

ns-3 modules implementing the LoRaWAN protocol are introduced in [2], [3], [4], [5]. An implementation of LoRaWAN in OMNET++ is described in [7]. In analysis, [7] models the Adaptive Data Rate algorithm, [2] simulates dense environments, [4] focuses on scalability when downlink feedback is required, and [5] evaluates the impact of Carrier Sense in LoRaWAN. [3] and [5] do integrate an energy consumption model into their modules, but these are based on a simplified state machine, a single TX power, and the subset of current consumption values available in the datasheet of the SX1272.

\section{B. Energy Modeling in ns-3}

A framework providing a general method of implementing energy models in ns-3 was introduced in [8]. The framework separates the modeling of energy consumption into components for expenditure and sources, with standard interfaces then enabling interaction. An entirely modular energy consumption framework is an inherently abstracted approach to profiling the energy consumption of a real device [9], but nevertheless provides an accurate depiction of the energy consumption of individual components [8], and is also the sole methodology for energy model integration that has been accepted into the official ns-3 repositories. This framework was initially used to create energy models for $\mathrm{Wi}-\mathrm{Fi}$, and has 
since been applied to LTE [10] and IEEE 802.15.4 [11]. Our contribution is to apply this full methodology to LoRaWAN.

\section{Energy Efficiency in LoRaWAN}

An analytical model of the energy consumption of the MultiTech mDot has been developed and used to make device lifetime estimates for a Class A LoRaWAN device [6]. However this research profiles the consumption of the whole device, and so the measurements are not suitable as input to an energy model developed using the framework described in [8]. The energy efficiency of Class A devices has also been calculated based on the use of the Channel Activity Detection mode while sending unacknowledged packets [12], with the model then used to calculate the expected lifetime of a device based on the transmission parameters. In previous research, hardware measurements have been taken as input to models, with results calculated analytically. Our research is the first to take a full measurement of readings for a LoRa transmitter and use it to develop a module for use in general simulation.

\section{ReAl World MeAsurements}

In this section, we perform an analysis of the energy consumption of the SX1272 transceiver in each possible state to derive a consumption profile beyond the subset of figures provided in the datasheet [13]. Our setup is an RFLORA-868-SO from RF Solutions with an integrated SX1272, mounted onto an atmega128RFA1 microcontroller. The current consumption was measured across the VCC and GND of the RF board (i.e. just the transceiver) using an Keithley 2000 multimeter, with the power of each transmission measured using a HP 8595E Spectrum Analyzer connected to the ANT pin of the RF board. The software on the microcontroller was a modified version of LMIC integrated into Contiki OS. The transmission settings are those outlined in Table I.

TABLE I

DEFAULT VALUES FOR MEASUREMENTS

\begin{tabular}{|l|l|}
\hline Setting & Value \\
\hline Supply Voltage & $3.3 \mathrm{~V}$ \\
\hline Frequency & channel hopping in $868 \mathrm{MHz}$ band \\
\hline LoRaWAN Data Rate & 0 \\
\hline Forward Error Correction & $4 / 5$ \\
\hline CRC & Enabled \\
\hline Payload length & 51 bytes \\
\hline Preamble Length & 8 symbols \\
\hline Temperature & $22{ }^{\circ} \mathrm{C}$ \\
\hline
\end{tabular}

The SX1272 defines a set of operating modes which can be selected via SPI to control the device's behaviour [13]. The current consumption observed in each of these modes corresponds to the set of states listed in Table II (FSK/OOKonly states are not included). An analysis of these states is thus performed to build a device energy consumption profile.

1) LoRa states without config. parameters: The current consumption for the 3 initial states outlined in Table II, which have no further config. parameters, are outlined in Table III.
TABLE II

SX1272 MODES AND STATES

\begin{tabular}{|l|l|}
\hline Device Mode & Consumption State \\
\hline Sleep & Sleep \\
\hline Standby & Standby \\
\hline Freq. Synthesis TX & Synthesizer (TX) \\
\hline Freq. Synthesis RX & Synthesizer (RX) \\
\hline TX & TX \\
\hline Channel Activity Detection & RX \\
\hline RXCONTINUOUS & RX \\
\hline RXSINGLE & RX \\
\hline
\end{tabular}

TABLE III

SX1272 Other States CuRrent Measurements

\begin{tabular}{|l|l|}
\hline State & Typ (mA) \\
\hline Sleep & $10^{-3}$ \\
\hline Standby & $1.6 \pm 0.042$ \\
\hline Synthesizer (TX) & $5.7 \pm 0.154$ \\
\hline Synthesizer (RX) & $5.0 \pm 0.145$ \\
\hline
\end{tabular}

2) $R X$ Current Consumption: The SX1272 provides an LNA (Low Noise Amplifier) boost option, which improves performance in RX mode while increasing current consumption. Values for both configurations are given in Table IV.

TABLE IV

SX1272 RX CURRENT MEASUREMENTS

\begin{tabular}{|l|l|l|}
\hline State & Bandwidth (kHz) & Typ (mA) \\
\hline With LNA Boost & 125 & $11 \pm 0.014$ \\
\hline With LNA Boost & 250 & $12 \pm 0.017$ \\
\hline Without LNA Boost & 125 & $10 \pm 0.015$ \\
\hline Without LNA Boost & 250 & $11 \pm 0.023$ \\
\hline
\end{tabular}

3) TX Current Consumption: There are two configurations for TX power selection on the SX1272 connected to two different output pins, and as such the choice of block must be made both in hardware and software. These are:

1) RFO pin - connected to the RFO pin is the unregulated single low power amplifier PA0, which can deliver an output power of between -1 and $+14 \mathrm{dBm}$, in $1 \mathrm{~dB}$ steps.

2) PA_BOOST pin - connected to the PA_BOOST pin are the internally regulated PA1 and PA2 power amplifiers, which enable an output RF power of between +2 and $+17 \mathrm{dBm}$, in $1 \mathrm{~dB}$ steps, as well as a High Power Operation (HPO) mode of $+20 \mathrm{dBm}$.

As the particular LoRa module we use, the RF-LORA-868SO, only provides an output to the PA_BOOST pin, a set of values for that configuration are given only, in Table V.

\section{Device Energy Model Class in NS-3}

The design of the LoRaWAN Energy Model follows the framework from [8]. The LoRaWANEnergyModel class subtracts energy from the node's energy source when the state of the node's PHY layer changes, with the depletion based on the length of time the node was in the previous state. The core current consumption functions of the node are contained within a LoRaWANCurrentModel object inside the LoRaWANEnergyModel. The actual consumption values are 
TABLE V

SX1272 TX CURRENT MEASUREMENTS

\begin{tabular}{|l|l|l|l|}
\hline State & Typ (mA) & State & Typ (mA) \\
\hline PA_Boost + HPO & & PA_Boost & \\
\hline$+20 \mathrm{dBm}$ & $105 \pm 0.812$ & $+10 \mathrm{dBm}$ & $42 \pm 0.171$ \\
\hline PA_Boost & & $+9 \mathrm{dBm}$ & $40 \pm 0.131$ \\
\hline$+17 \mathrm{dBm}$ & $77 \pm 0.475$ & $+8 \mathrm{dBm}$ & $39 \pm 0.100$ \\
\hline$+16 \mathrm{dBm}$ & $69 \pm 0.526$ & $+7 \mathrm{dBm}$ & $37 \pm 0.074$ \\
\hline$+15 \mathrm{dBm}$ & $62 \pm 0.809$ & $+6 \mathrm{dBm}$ & $36 \pm 0.060$ \\
\hline$+14 \mathrm{dBm}$ & $54 \pm 0.408$ & $+5 \mathrm{dBm}$ & $35 \pm 0.093$ \\
\hline$+13 \mathrm{dBm}$ & $50 \pm 0.462$ & $+4 \mathrm{dBm}$ & $34 \pm 0.037$ \\
\hline$+12 \mathrm{dBm}$ & $47 \pm 0.284$ & $+3 \mathrm{dBm}$ & $33 \pm 0.039$ \\
\hline$+11 \mathrm{dBm}$ & $44 \pm 0.219$ & $+2 \mathrm{dBm}$ & $32 \pm 0.016$ \\
\hline
\end{tabular}

defined in the SX1272LoRaWANCurrentModel class, which inherits from the abstract LoRaWANCurrentModel class and contains the readings described in Section III. This approach enables the easy implementation of other models in the future (e.g., for the SX1276). The only required changes to the model to integrate it into a LoRaWAN module are the links to the PHY layer of each implementation, which is limited to 2 model variables and the PHY layer traced values. This integration has been performed for 3 modules: $[2]^{1},[3]^{2},[4]^{3}$.

\section{Simulations}

To judge the quality of the model, the energy model is used to calculate the average current consumption of the regular transmission of unacknowledged LoRaWAN packets for each data rate. These results are then compared to equivalent results derived from the analytical model introduced in [6].

\section{A. Adaption of analytical model}

As the energy model only models the transceiver, the analytical model has to be modified to remove device-related states. As in the original model, the average current consumption is:

$$
I_{\text {avg }}=\frac{Q_{\text {packet }}}{T_{\text {period }}}
$$

where $T_{\text {period }}$ is the time between two consecutive transmissions. However, differently from [6], the charge, $Q_{\text {packet }}$ is calculated using only transceiver-related terms:

$$
\begin{aligned}
Q_{\text {packet }}= & \left(T_{t x} * I_{t x}\right)+\left(T_{w w 1} * I_{\text {std }}\right)+\left(T_{r x 1} * I_{r x}\right) \\
& +\left(T_{w w 2} * I_{s t d}\right)+\left(T_{r x 2} * T_{r} r x 2\right)+\left(T_{\text {sleep }} * I_{\text {sleep }}\right)
\end{aligned}
$$

Each of the time-related terms of $Q_{\text {packet }}$ have been calculated according to the equations described in [6], and the measurements described above have been used as input to the current-related terms. The maximum TX power available in the majority of LoRaWAN channels in the EU sub-1GHz ISM bands has been chosen as the value for $I_{t x}$ (i.e. $14 \mathrm{dBm}$ ).

\footnotetext{
${ }^{1}$ https://github.com/ConstantJoe/signtlabdei-lorawan-with-energy-model

${ }^{2}$ https://github.com/ConstantJoe/ku-leuven-lorawan-with-energy-model

${ }^{3} \mathrm{https}: / /$ github.com/ConstantJoe/imec-idlab-lorawan-with-energy-model
}

\section{B. Simulations}

The energy model was integrated into the module from [4]. Simulations consist of a network with 1 Network Server, 1 Gateway, and 10 devices placed within a Gateway-centred disk with a radius of $1 \mathrm{~km}$. Each device sends 1 unacknowledged Class A LoRaWAN packet randomly within each $T_{\text {period }}$. Each simulation was run for 1 day, and the average current consumption of each device was calculated as:

$$
I_{\text {avg }}=\frac{\left(E_{\text {Source_Initial }}-E_{\text {Source_Final }}\right) / V c c}{T_{\text {day }}}
$$

\section{Results}

The adapted analytical model and the simulator were then used to generate the average current consumption for each data rate, for an increasing transmission period. Figs. 2 and 3 show the average current consumption achieved as a function of the data rate and $T_{\text {period }}$, with Fig. 2 showing the theoretical limits calculated using the analytical model, and Fig. 3 showing the results generated using the simulator. Figs. 4 and 5 show a focus on the latter ends of the curves.

The sole difference between the results is in the simulations with the lowest transmission periods. In the simulator, transmissions inside the duty cycle of a subband are prevented, and so there is a limit in time across all subbands in which a device can be transmitting. Since in LoRaWAN Class A receive windows only follow transmissions, there is a maximum "active time" in LoRaWAN for a device in a set period, beyond which it must remain in sleep mode. This manifests in the graph in the hard limit reached (at $0.6 \mathrm{~mA}$ ). As the analytical model does not factor in the duty cycle limit, results differ when these limits are breached. Beyond this, there is no deviation. This shows that the module does model the LoRaWAN protocol.

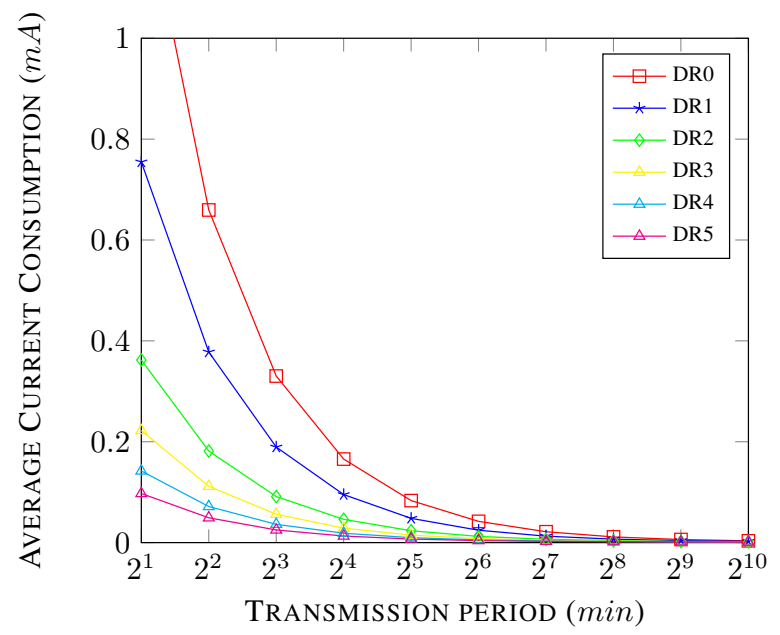

Fig. 1. Average current consumption - theoretical limit

\section{CONCLUSIONS}

In this research, an analysis of the energy consumption of different states of the SX1272 was performed. The results 


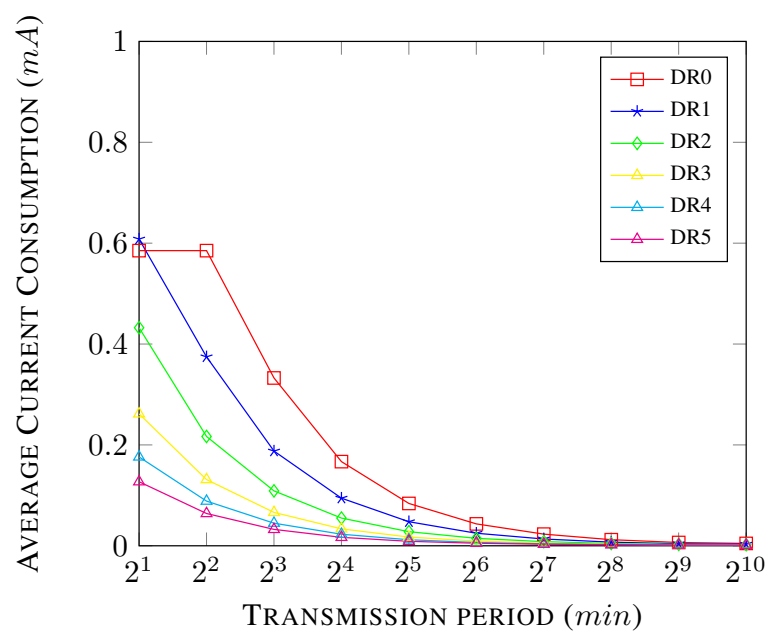

Fig. 2. Average current consumption - ns-3 simulation

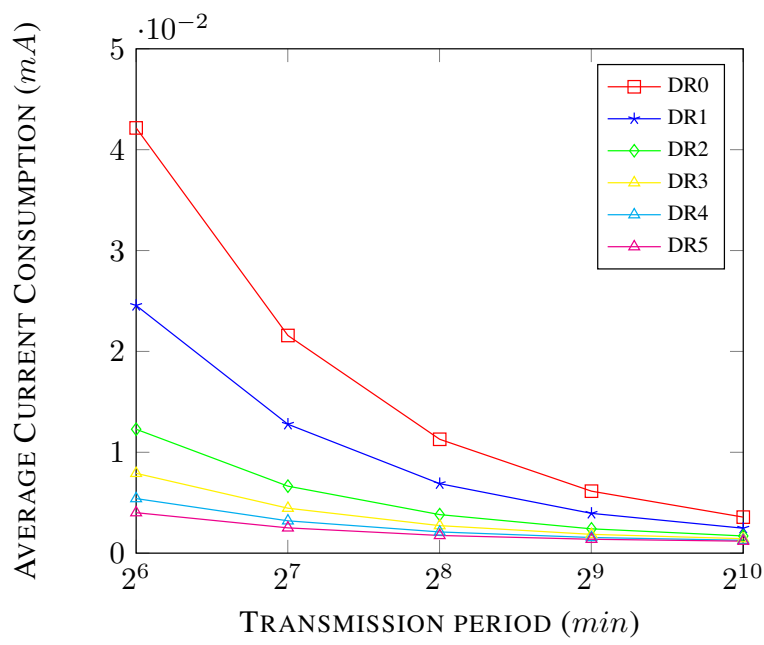

Fig. 3. Average current consumption - theoretical limit (focus)

were used to develop a LoRaWAN energy model for use in ns-3, integrated into three of the current publicly available LoRaWAN ns-3 modules. An evaluation of the model through comparisons with the analytical model developed in [6] was performed. The results show that the model does successfully simulate LoRaWAN Class A energy consumption.

Future work will involve a comparison of energy consumption of Class A and Class B in simulation using the model developed here. We also intend to build an energy consumption profile of the SX1272 with use of the RFO pin, as well as include temperature as a factor in the model. In addition, the modeling of the full LoRaWAN protocol in ns-3 should be undertaken, to enable the evaluation of realistic devices in terms of energy efficiency and scalability through simulation.

\section{ACKNOWLEDGMENT}

This publication has emanated from research conducted with the financial support of Science Foundation Ireland (SFI)

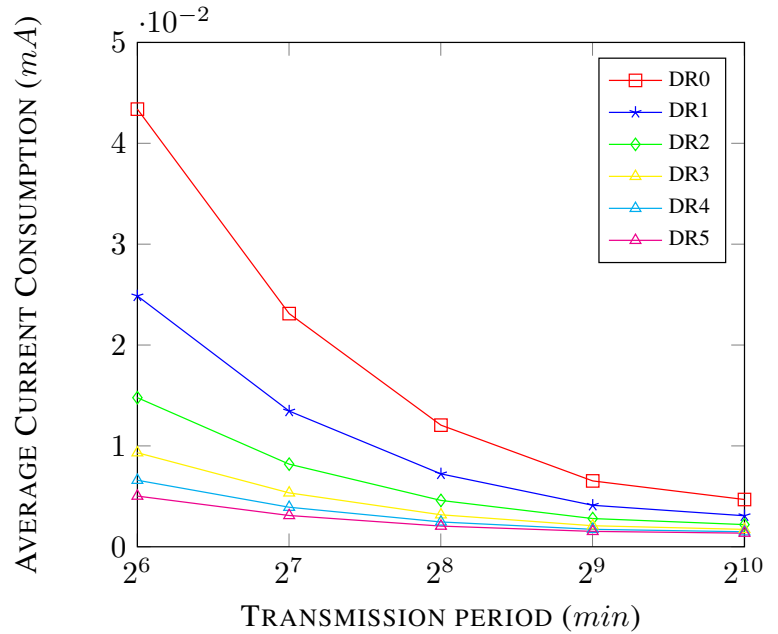

Fig. 4. Average current consumption - ns-3 simulation (focus)

and is co-funded under the European Regional Development Fund under Grant Number 13/RC/2077.

\section{REFERENCES}

[1] U. Raza, P. Kulkarni, and M. Sooriyabandara. Low Power Wide Area Networks: An overview. IEEE Communications Surveys Tutorials, PP(99):1-1, 2017.

[2] D. Magrin, M. Centenaro, and L. Vangelista. Performance evaluation of LoRa networks in a smart city scenario. In 2017 IEEE International Conference on Communications (ICC), pages 1-7, May 2017.

[3] B. Reynders, Q. Wang, and S. Pollin. A LoRaWAN module for NS-3: Implementation and evaluation. In Proceedings of the 10th Workshop on Ns-3, WNS3 '18, pages 61-68, New York, NY, USA, 2018. ACM.

[4] F. Van den Abeele, J. Haxhibeqiri, I. Moerman, and J. Hoebeke. Scalability analysis of large-scale LoRaWAN networks in NS-3. IEEE Internet of Things Journal, 4(6):2186-2198, Dec 2017.

[5] T. To and A. Duda. Simulation of LoRa in NS-3: Improving LoRa performance with CSMA. In 2018 IEEE International Conference on Communications (ICC), pages 1-7, May 2018.

[6] L. Casals, B. Mir, R. Vidal, and C. Gomez. Modeling the energy performance of LoRaWAN. Sensors, 17(10), 2017.

[7] M. Slabicki, G. Premsankar, and M. Di Francesco. Adaptive configuration of LoRa networks for dense IoT deployments. In 2018 IEEE/IFIP Network Operations and Management Symposium (NOMS), 2018.

[8] H. Wu, S. Nabar, and R. Poovendran. An energy framework for the Network Simulator 3 (NS-3). In Proceedings of the 4th International ICST Conference on Simulation Tools and Techniques, SIMUTools '11, pages 222-230, 2011.

[9] A. Garcia-Saavedra, P. Serrano, A. Banchs, and G. Bianchi. Energy consumption anatomy of 802.11 devices and its implication on modeling and design. In Proceedings of the 8th International Conference on Emerging Networking Experiments and Technologies, CoNEXT'12, pages 169-180, New York, NY, USA, 2012. ACM.

[10] S. T. V. Pasca, B. Akilesh, A. V. Anand, and B. R. Tamma. A NS-3 module for LTE UE energy consumption. In 2016 IEEE International Conference on Advanced Networks and Telecommunications Systems (ANTS), pages 1-6, Nov 2016.

[11] V. Rege and T. Pecorella. A realistic MAC and energy model for 802.15.4. In Proceedings of the Workshop on Ns-3, WNS3 '16, pages 79-84, New York, NY, USA, 2016. ACM.

[12] M. Costa, T. Farrell, and L. Doyle. On energy efficiency and lifetime in Low Power Wide Area Network for the Internet of Things. In 2017 IEEE Conference on Standards for Communications and Networking (CSCN), pages 258-263, Sept 2017.

[13] Semtech. SX1272/73 - $860 \mathrm{MHz}$ to $1020 \mathrm{MHz}$ low power long range transceiver Rev. 3.1, 32017. 